\title{
Association of Vital Exhaustion with Risk Factors for Cardiovascular Diseases, Quality of Life and Lifestyle in 41-44-Year-Old Muscovite Men
}

\author{
Marina B. Kotova *, Vyacheslav B. Rozanov (D), Anton R. Kiselev (D), Sergey A. Maksimov (DD \\ and Oxana M. Drapkina 1
}

check for

updates

Citation: Kotova, M.B.; Rozanov, V.B.; Kiselev, A.R.; Maksimov, S.A.; Drapkina, O.M. Association of Vital Exhaustion with Risk Factors for Cardiovascular Diseases, Quality of Life and Lifestyle in 41-44-Year-Old Muscovite Men. Int. J. Environ. Res. Public Health 2021, 18, 9691. https:/ / doi.org/10.3390/ijerph18189691

Academic Editor: Mirja Hirvensalo

Received: 7 July 2021

Accepted: 12 September 2021

Published: 14 September 2021

Publisher's Note: MDPI stays neutral with regard to jurisdictional claims in published maps and institutional affiliations.

Copyright: (C) 2021 by the authors Licensee MDPI, Basel, Switzerland. This article is an open access article distributed under the terms and conditions of the Creative Commons Attribution (CC BY) license (https:// creativecommons.org/licenses/by/ $4.0 /$ )
National Medical Research Center for Therapy and Preventive Medicine, 101990 Moscow, Russia; vbrozanov@gmail.com (V.B.R.); antonkis@list.ru (A.R.K.); SMaksimov@gnicpm.ru (S.A.M.); drapkina@bk.ru (O.M.D.)

* Correspondence: mkotova@gnicpm.ru

Abstract: (1) Background: Vital exhaustion (VE) is no less of an important risk factor (RF) for cardiovascular diseases (CVD) and cardiovascular events than the well-known RFs. Insufficient knowledge of the relationship between VE and CVD RF, quality of life, and lifestyle was the rationale for this study. (2) Methods: We examined 301 Muscovite men 41-44 years of age. The categorization of RFs for CVD was carried out in accordance with conventionally considered criteria. In order to evaluate the lifestyle and quality of life in study participants, we were offering them a self-filling questionnaire developed by I.A. Gundarov. The presence of VE signs was assessed using a 14-item short version of the Maastricht Vital Exhaustion Questionnaire scale (MVEQ). All study subjects were classified into three ordered groups depending on the distribution of VE indicators by tertiles: Group 1 consisted of men with a low VE (0-2 points), Group 2 included males with a medium VE score (3-5 points), and Group 3 comprised subjects with high VE scores (6-14 points). To analyze the obtained data, we used one-way analysis of variance (ANOVA), Pearson's chi-squaredtest $\left(\chi^{2}\right)$, Goodman and Kruskal's gamma, and linear regression analysis. (3) Results: We established that every third male (36.8\%) had VE signs, while $10.6 \%$ of men had high VE levels. With an increase of VE in men, the frequency of arterial hypertension $(\mathrm{AH})$ was increasing as well, and it was significantly higher in men with a high VE compared to their peers with a low VE (48.4\% versus $33 \%$; $p=0.03)$. A significant linear relationship was discovered between VE levels and excessive alcohol consumption $(p=0.001)$. The strongest linear associations were found between the VE level, and both psychosocial stress indicator and the amount of consumed ethanol. Self-assessment of personal happiness, job and sleep satisfaction, residential living conditions, and spiritual needs, as well as psychosocial stress indicator, total amount of consumed ethanol, and muscle strength (hand-grip dynamometry), were independent determinants of the VE level, and, collectively, they explained $46.6 \%$ of its variability. The greatest contribution to VE was made by the personal happiness level, explaining $25.5 \%$ of its variability. The proportions of the VE variance uniquely explained by various factors were as follows: $9.3 \%$ by the psychosocial stress, $4.9 \%$ by job satisfaction, $2.8 \%$ by sleep satisfaction, $2.3 \%$ by total consumption of ethanol, $1.6 \%$ by muscle strength, $1.1 \%$ by living conditions in the residential neighborhood, and just $0.8 \%$ by spiritual needs. (4) Conclusion: High VE levels in 41-44-year-old men are associated with $\mathrm{AH}$, sedentary behavior, excessive alcohol consumption, and lower values of most indicators of both lifestyle and quality of life.

Keywords: vital exhaustion; psychosocial stress; cardiovascular diseases; hypertension; physical activity; alcohol; hand-grip dynamometry; lifestyle; quality of life

\section{Introduction}

Psychosocial risk factors (RFs), along with conventional RFs, increase the risk of developing cardiovascular diseases (CVD), impair CVD prognosis, and prevent strong adherence 
to treatment and efforts to improve lifestyle, both in patients and in the population as a whole [1]. According to the European Guidelines on Cardiovascular Disease Prevention in Clinical Practice [1], psychological factors include parameters caused primarily by stressful impacts: anxiety, depression, hostility, vital exhaustion (VE), and sleep disorders [1].

In their meta-analysis, Cohen et al. (2017) showed that VE is a RF for CVD that is no less important than other conventionally accepted factors [2].

The term 'vital exhaustion'was first introduced in 1987 by Appels et al. in a prospective Rotterdam Civil Servants Study. VE was defined as feeling excessively tired, exhausted, irritable, and demoralized [3]. Over the past decade, VE has attracted considerable attention from researchers due to its adverse effects on health and well-being [4-6]. In particular, the conducted studies have shown that VE was an independent RF for the development and progression of CVD [2,4-7], and men were at greater risk of developing CVD than women $[8,9]$. At present, VE is an understudied CVD RF. It is an early indication of acute myocardial infarction and an RF for coronary heart disease and cerebrovascular events [2,6,10-12]. The cause of VE is not fully understood as yet. It is assumed that VE develops as a result of a breakdown in adaptation to chronic stress [8,13]. People experiencing periodic or prolonged stress are more likely to exhibit unhealthy behavior, such as undereating or overeating, low physical activity, and alcohol abuse, which, in turn, negatively affects their health [14]. Some studies suggested that poor health was associated with social and occupational stress, low social support, and VE [15].

Consequently, the adverse impact of VE on human health and well-being, as well as its relationship with CVD, can be mediated by changes in lifestyle and deterioration of the quality of life.

Hence, currently, there is a dire need for studying VE in human populations and its impact on CVD morbidity and prognosis. In particular, insufficient knowledge of the relationship between VE and CVD RFs, quality of life, and lifestyle served was the rationale for this study.

The objective of our research was to evaluate the associations of VE with CVD RFs, lifestyle, and quality of life in 41-44-year-old Muscovite men.

\section{Materials and Methods}

\subsection{Study Participants}

In 1983, a sample was formed for a long-term prospective observational study on the dynamics of main CVD RFs. The sample included fifth grade school students residing in Moscow. At baseline, 23 out of 79 schools were randomly selected. The chosen schools enrolled 1182 boys, 11-12 years of age, attending the fifth grade. A total of 1005 individuals were examined, which constituted $85 \%$ of the selected population. Their average age was $11.9 \pm 0.11$ years. Over 32 years of prospective study, 7 medical examinations were carried out at different intervals. Our study participants were represented by men selected for subsequent analysis at their seventh visit to a physician within the framework of the above-mentioned prospective study.

\subsection{Measurement Methods}

The procedure of medical examination encompassed the following components: a standard questionnaire with passport information, educational status, social status, personal anamnesis, family medical history, lifestyle (physical activity, cigarette smoking, and alcohol consumption); threefold measurement of blood pressure (BP); measurements of body mass (BM) and height, as well as of waist and hip circumference (WC, HC); and determination of the levels of total cholesterol, high density lipoprotein cholesterol (HDL cholesterol), triglycerides, and low density lipoprotein cholesterol (LDL cholesterol). The body mass index (BMI) was used to evaluate body mass excess (BME) and obesity, and the ratio of WC to HC was calculated to characterize an abdominal obesity.

The arterial hypertension $(\mathrm{AH})$ group included men with a $\mathrm{BP} \geq 140 / 90 \mathrm{mmHg}$, as well as with a $\mathrm{BP}<140 / 90 \mathrm{mmHg}$ but receiving antihypertensive treatment. BME was 
characterized by BMI $25 \geq \mathrm{kg} / \mathrm{m}^{2}$, whereas obesity was categorized at BMI $\geq 30 \mathrm{~kg} / \mathrm{m}^{2}$. The group of abdominal obesity included subjects with $W C \geq 94 \mathrm{~cm}$. The risk categories for the blood lipid panel were formed on the basis of borderline high values sensu, the classification presented in the third report of the expert group of the National Cholesterol Education Program (NCEP) on detection, assessment, and therapy of high cholesterol levels in adults-Adult Treatment Panel III (ATP III) [16].

The presence of VE signs was established via a 14-item short version of the Maastricht Vital Exhaustion Questionnaire scale (MVEQ) [3]. The Maastricht Questionnaire includes 14 items that describe various aspects of VE with a possible score range from 0 to 14 points. In order to ensure comparability and sufficient sample sizes in the compared groups, all study participants were classified into three ordered groups depending on the distribution of VE indicators by tertiles. Group 1 consisted of men with a low VE (0-2 points), Group 2 included males with a medium VE score (3-5 points), and Group 3 comprised the subjects with high VE values (6-14 points).

The Reeder Stress Inventory (L.G. Reeder et al. 1969), adapted by O.S. Kopina et al. (1989), was used as the method of express diagnosing of the psychosocial stress (PS) level [17].

To evaluate the lifestyle and quality of life in the study participants, we were offering study subjects a self-filling questionnaire developed by I.A. Gundarov [18]. The following parameters were analyzed: material well-being in a household; personal earnings; living conditions of a subject; environmental conditions in the area of a subject's residence; family and children; variety of a consumer food basket and satisfaction with food; love and sexual feelings; spiritual needs, social support and communication with friends; and the type of work and position in society. The quality-of-life indicators were assessed by the respondents on a 100-point scale.

In addition to the I.A. Gundarov's questionnaire, such behavioral habits as smoking, alcohol consumption, and physical activitylevelwere assessed to identify the lifestyle of each study participant.

To measure physical activity, the International Physical Activity Questionnaires (IPAQ) were employed [19]. Physical activity categories were formed in accordance with IPAQ recommendations [20]. Men smoking at least one cigarette a day were considered regular smokers. To assess alcohol consumption, we used standard questionnaires borrowed from the Russian Longitudinal Monitoring Survey (RLMS) [21] and the Survey on Stress, Aging and Health in Russia (SAHR) [22]. Questions included the following items: frequency and volume of alcohol consumption (per day, per week, per month) throughout the year, and categorization of alcoholic beverages (beer, wine, spirits, etc.). Alcohol consumption has been converted to pure ethanol, and excessive alcohol use was stated for those who consumed over $168 \mathrm{~g}$ of pure ethanol per week.

\subsection{Statistical Analyses}

Descriptive statistics presented in tables and a figure have the following notations: $\mathrm{n}$ is the sample size (number of subjects) in a group; \% represents the proportion of subjects of their total number in a group; $\mathrm{M}$ is an arithmetic mean; and 95\% CI denotes a $95 \%$ confidence interval. To check quantitative variables for normality of distribution, we used descriptive statistics, histograms of residuals, and normal probability plots (Q-Q plots). The homogeneity of variances was checked using the Levene's test. One-way analysis of variance (ANOVA) was used to test for a linear association among the ordered categories of the factor variable and dependent continuous variables. The Holm-Bonferroni correction was applied to eliminate a power drop with a large number of dependent variables. Group comparisons, corrected for differences in one or more variables, were performed using the least squares method in the SAS PROC GLM procedure. The hypothesis on dependence of the distribution of subjects with CVD risk factors on the magnitude of VE was tested using the Pearson's chi-squared test $(\chi 2)$, followed by pairwise comparisons of proportions via Z-test. The strength and direction of the relationship between ordinal and 
dichotomous variables was evaluated by means of Goodman and Kruskal's gamma test (gamma coefficient). The association of VE with the studied parameters was assessed using multiple linear regression with stepwise input of independent variables into the model. The variance inflation factor (VIF) was used to check for collinearity.

The critical level of statistical significance $(p)$ in our study was 0.05 . Statistical data processing was performed using SAS (Statistical Analysis Software) 9.0 and IBM SPSS Statistics version 23 software for Windows.

\section{Results}

After 32 years, out of 1005 males, just 301 (30\%) representatives of the original population sample were examined on their seventh medical examination visit. The average age of surveyed men at the moment was 42.9 years.

We estimated the frequency of different VE parameters in the surveyed sample of 41-44-year-old men using unambiguous criteria [3]. In particular, $26.2 \%$ of surveyed men were characterized by a medium VE level, whereas $10.6 \%$ had high VE scores (Figure 1).

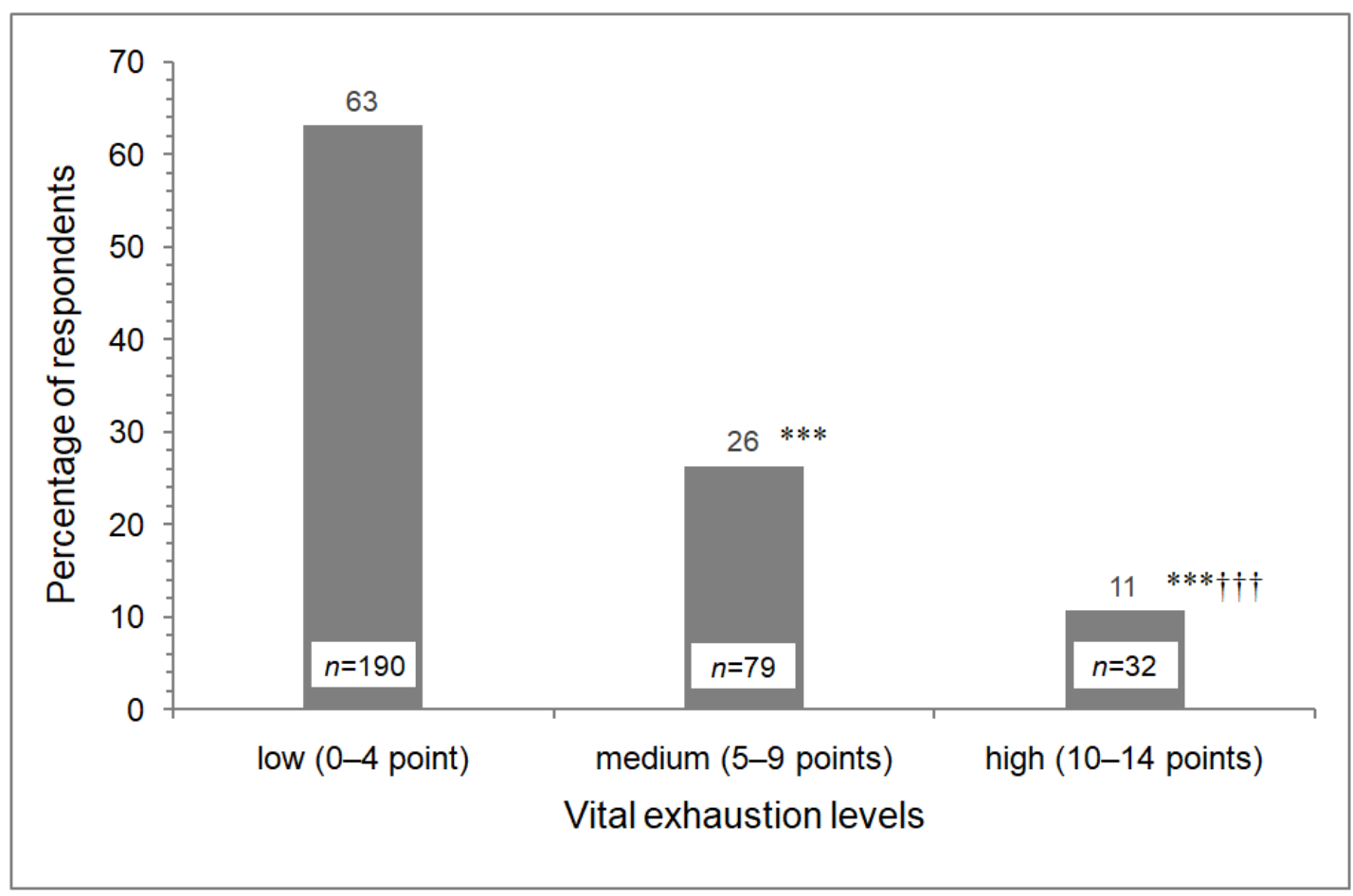

Figure 1. Distribution of male respondents among the vital exhaustion levels (1-low, 2-medium, 3-high) $(N=301)$. Unambiguous criteria were chosen to categorize the vital exhaustion. ${ }^{* * *} p<0.001$ versus level 1 ; $t^{*}+p<0.001$ versus level 2. Paired comparisons are made using Z-criteria with Holm-Bonferroni correction.

The frequency distribution of CVD RFs, according to ordered VE levels, is presented in Table 1. This table suggests the relationship between AH and VE level (Pearson's $\chi^{2}=6.043, p=0.049$ ) and association among excessive alcohol use and VE level (Pearson's $\left.\chi^{2}=12.155, p=0.002\right)$. These dependences are linear $\left(\chi^{2}\right.$ for trend $=4.693, p=0.030 ; \chi^{2}$ for trend $=11.946, p=0.001$, respectively), i.e., with increase in VE level, there is a rise in both $\mathrm{AH}$ frequency and frequency of excessive alcohol consumption in 41-44-year-old men. AH incidence was statistically significantly higher among men with a high VE compared with low and medium VE levels, and the frequency of excessive alcohol use was higher among men with medium and high VE levels versus low VE level. The established a directly proportional relationship between VE level and indicated RFs wereweak for $\mathrm{AH}$ (gamma $=0.193 ; p=0.045)$ and moderately strong for excessive alcohol use (gamma $=0.385$; $p<0.001)$. 
Table 1. Distribution of risk factors in groups of males with different vital exhaustion levels.

\begin{tabular}{|c|c|c|c|c|c|c|}
\hline \multirow{2}{*}{ Risk Factor } & & \multicolumn{3}{|c|}{ Vital Exhaustion Levels } & \multicolumn{2}{|c|}{ Chi-Squared Tests } \\
\hline & & $\begin{array}{c}1 \\
(n=97)\end{array}$ & $\begin{array}{c}2 \\
(n=109)\end{array}$ & $\begin{array}{c}3 \\
(n=93)\end{array}$ & $\begin{array}{l}\text { Linear-by-Linear } \\
\text { Association }\end{array}$ & $\begin{array}{l}\text { Pearson's } \\
\text { Chi-Square }\end{array}$ \\
\hline \multirow{2}{*}{$\begin{array}{l}\text { Overweight status }+ \\
\text { obesity }\end{array}$} & yes & $71(73)$ & $73(67)$ & $57(61)$ & \multirow{2}{*}{$\begin{array}{l}x^{2}=3.05 \\
p=0.081\end{array}$} & \multirow{2}{*}{$\begin{array}{c}x^{2}=3.06 \\
p=0.26\end{array}$} \\
\hline & no & $26(27)$ & $36(33)$ & $36(39)$ & & \\
\hline \multirow{2}{*}{ Abdominal obesity } & yes & $50(52)$ & $54(50)$ & $40(43)$ & \multirow{2}{*}{$\begin{array}{l}x^{2}=1.37 \\
p=0.242\end{array}$} & \multirow{2}{*}{$\begin{array}{l}x^{2}=1.52 \\
p=0.468\end{array}$} \\
\hline & no & $47(48)$ & $55(50)$ & $53(57)$ & & \\
\hline \multirow{2}{*}{ Arterial hypertension } & yes & $32(33)$ & $37(34)$ & $45(48)^{*}+$ & \multirow{2}{*}{$\begin{array}{l}x^{2}=4.69 \\
p=0.030\end{array}$} & \multirow{2}{*}{$\begin{array}{l}x^{2}=6.04 \\
p=0.049\end{array}$} \\
\hline & no & $65(67)$ & $72(66)$ & $48(52)$ & & \\
\hline \multirow{2}{*}{ Dyslipidemia } & yes & $73(75)$ & $83(76)$ & $65(70)$ & \multirow{2}{*}{$\begin{array}{l}x^{2}=0.69 \\
p=0.405\end{array}$} & \multirow{2}{*}{$\begin{array}{l}x^{2}=1.15 \\
p=0.562\end{array}$} \\
\hline & no & $24(25)$ & $26(24)$ & $28(30)$ & & \\
\hline \multirow{2}{*}{ Current smokers } & yes & $40(41)$ & $50(46)$ & $44(47)$ & \multirow{2}{*}{$\begin{array}{l}x^{2}=0.71 \\
p=0.399\end{array}$} & \multirow{2}{*}{$\begin{array}{l}x^{2}=0.79 \\
p=0.675\end{array}$} \\
\hline & no & $57(59)$ & $59(54)$ & $49(53)$ & & \\
\hline \multirow{2}{*}{ Low physical activity } & yes & $27(28)$ & $24(22)$ & $27(29)$ & \multirow{2}{*}{$\begin{array}{l}x^{2}=0.03 \\
p=0.863\end{array}$} & \multirow{2}{*}{$\begin{array}{l}x^{2}=1.51 \\
p=0.471\end{array}$} \\
\hline & no & $70(72)$ & $85(78)$ & $66(71)$ & & \\
\hline \multirow{2}{*}{ Alcohol consumption } & yes & $76(78)$ & $86(79)$ & $80(86)$ & \multirow{2}{*}{$\begin{array}{l}x^{2}=1.78 \\
p=0.182\end{array}$} & \multirow{2}{*}{$\begin{array}{l}x^{2}=2.27 \\
p=0.321\end{array}$} \\
\hline & no & $21(22)$ & $23(21)$ & $13(14)$ & & \\
\hline \multirow{2}{*}{$\begin{array}{l}\text { Excessive alcohol } \\
\text { consumption }\end{array}$} & yes & $11(11)$ & $26(24) *$ & $30(32)^{* * *}$ & \multirow{2}{*}{$\begin{array}{c}x^{2}=11.95 \\
p=0.001\end{array}$} & \multirow{2}{*}{$\begin{array}{c}\mathrm{x}^{2}=12.16 \\
p=0.002\end{array}$} \\
\hline & no & $86(89)$ & $83(76)$ & $63(68)$ & & \\
\hline
\end{tabular}

Data are presented as $\mathrm{n}(\%)$.Hereinafter, the 3 groups are ordered according to the increasing level of vital exhaustion (VE): 1-low VE level;

2-medium VE level; 3-high VE level. Data are presented as $\mathrm{n}(\%) .{ }^{*} p<0.05,{ }^{* * *} p<0.001$ (versus level 1); $\uparrow p<0.05$ (versus level 2).

Paired comparisons are made using Z-criteria with Holm-Bonferroni correction.

Comparative analysis of the studied CVD risk markers in groups of men with different VE levels (Table 2) demonstrated statistically significant unidirectional linear trends of VE versus group average BMI, hand-grip dynamometry, HDL cholesterol, volume of consumed alcohol, duration of sedentary behavior, and PS level. The strongest linear relationship was established between VE level and PS values.

Table 2. Comparative analysis of CVD risk markers in groups of males with different vital exhaustion levels.

\begin{tabular}{|c|c|c|c|c|}
\hline \multirow{2}{*}{ Studied Indicators } & \multicolumn{3}{|c|}{ Vital Exhaustion Levels } & \multirow{2}{*}{ F-Test for Trend } \\
\hline & $1(n=97)$ & $2(n=109)$ & $3(n=93)$ & \\
\hline Age, years & $42.9(42.8-43.0)$ & $43.0(42.9-43.1)$ & $42.9(42.8-43.0)$ & $\mathrm{F}=0.60, p=0.440$ \\
\hline $\mathrm{SBP}, \mathrm{mmHg}$ & $121(118-123)$ & $122(119-124)$ & $125(121-128)$ & $\mathrm{F}=3.17, p=0.076$ \\
\hline $\mathrm{DBP}, \mathrm{mmHg}$ & $82(80-84)$ & $82(80-84)$ & $84(81-86)$ & $\mathrm{F}=1.67, p=0.198$ \\
\hline Pulse, beats per minute & $73(71-75)$ & $75(73-77)$ & $75(72-77)$ & $\mathrm{F}=0.96, p=0.328$ \\
\hline BMI, $\mathrm{kg} / \mathrm{m}^{2}$ & $28.7(27.6-29.7)$ & $27.5(26.6-28.4)$ & $26.9(25.9-27.8)$ * & $\mathrm{F}=6.85, p=0.009$ \\
\hline $\mathrm{WC}, \mathrm{cm}$ & $96.3(93.5-99.1)$ & $93.8(91.4-96.3)$ & $93.3(90.8-95.9)$ & $\mathrm{F}=2.38, p=0.124$ \\
\hline $\mathrm{WC} / \mathrm{HC}$ & $0.93(0.92-0.95)$ & $0.93(0.91-0.94)$ & $0.93(0.92-0.95)$ & $\mathrm{F}=0.03, p=0.871$ \\
\hline Hand-grip dynamometry, kg & $45.5(44.2-46.8)$ & $43.6(42.0-45.2)$ & $41.1(39.5-42.6)^{* * *}$ & $\mathrm{~F}=16.41, p<0.001$ \\
\hline $\mathrm{TCH}, \mathrm{mmol} / \mathrm{L}$ & $5.7(5.5-6.0)$ & $5.7(5.4-5.9)$ & $5.8(5.5-6.0)$ & $\mathrm{F}=0.01, p=0.907$ \\
\hline $\mathrm{HDL} \mathrm{CH}, \mathrm{mmol} / \mathrm{L}$ & $0.95(0.90-1.01)$ & $0.99(0.93-1.04)$ & $1.07(0.98-1.15)$ * & $\mathrm{F}=5.72, p=0.017$ \\
\hline $\mathrm{LDL} \mathrm{CH}, \mathrm{mmol} / \mathrm{L}$ & $4.1(3.9-4.4)$ & $4.0(3.8-4.3)$ & $4.0(3.8-4.3)$ & $\mathrm{F}=0.57, p=0.450$ \\
\hline $\mathrm{TG}, \mathrm{mmol} / \mathrm{L}$ & $1.4(1.2-1.6)$ & $1.5(1.3-1.6)$ & $1.5(1.2-1.7)$ & $\mathrm{F}=0.34, p=0.559$ \\
\hline $\begin{array}{l}\text { Number of smoked } \\
\text { cigarettes per day }\end{array}$ & $15(12-17)$ & $18(15-21)$ & $18(15-21)$ & $\mathrm{F}=2.22, p=0.138$ \\
\hline $\begin{array}{l}\text { The amount of consumed } \\
\text { ethanol, g per week }\end{array}$ & $77.3(55.1-99.5)$ & $119.1(80.2-158.1)$ & $201.0(136.7-265.2)^{* * *}+$ & $\mathrm{F}=14.59, p<0.001$ \\
\hline
\end{tabular}


Table 2. Cont.

\begin{tabular}{|c|c|c|c|c|}
\hline \multirow{2}{*}{ Studied Indicators } & \multicolumn{3}{|c|}{ Vital Exhaustion Levels } & \multirow{2}{*}{ F-Test for Trend } \\
\hline & $1(n=97)$ & $2(n=109)$ & $3(n=93)$ & \\
\hline $\begin{array}{l}\text { Physical training and sports, } \\
\text { hours per week }\end{array}$ & $2.3(1.7-2.9)$ & $2.5(1.9-3.1)$ & $1.5(1.0-2.1)$ & $\mathrm{F}=3.19, p=0.075$ \\
\hline $\begin{array}{l}\text { Sedentary behavior, hours } \\
\text { per day }\end{array}$ & $6.3(5.7-6.8)$ & $7.8(7.1-8.5)^{* *}$ & $7.4(6.7-8.1)^{*}$ & $\mathrm{~F}=5.89, p=0.016$ \\
\hline Psychosocial stress score & $3.2(3.1-3.3)$ & $2.9(2.8-3.0)^{* * *}$ & $2.5(2.4-2.7)^{* * *}++\dagger$ & $\mathrm{F}=79.02, p<0.001$ \\
\hline
\end{tabular}

Data are presented as M (95\% confidence interval). CVD—cardiovascular diseases, SBP—systolic blood pressure, DBP—diastolic blood pressure, BMI-body mass index, WC—waist circumference, HC—hip circumference, TCH—-total cholesterol, HDL CH—high density lipoprotein cholesterol, LDL CH-low density lipoprotein cholesterol, TG-triglycerides. ${ }^{*} p<0.05,{ }^{* *} p<0.01,{ }^{* * *} p<0.001$ (versus level 1); $+p<0.05,++\dagger p<0.001$ (versus level 2). $p$-values are derived from ANOVA with post hoc comparisons using the Holm-Bonferroni method.

Previously identified intergroup differences in BMI and HDL cholesterol (Table 3) disappeared after adjusting the CVD RFs in terms of PS level.

Table 3. Comparative analysis of CVD risk markers in groups of males with different vital exhaustion levels after the correction for the psychosocial stress score.

\begin{tabular}{|c|c|c|c|c|}
\hline \multirow{2}{*}{ Studied Indicators } & \multicolumn{3}{|c|}{ Vital Exhaustion Levels } & \multirow{2}{*}{ F-Test } \\
\hline & $1(n=97)$ & $2(n=109)$ & $3(n=93)$ & \\
\hline $\mathrm{SBP}, \mathrm{mmHg}$ & $121(118-124)$ & $122(119-124)$ & $125(121-128)$ & $\mathrm{F}=1.58, p=0.208$ \\
\hline $\mathrm{DBP}, \mathrm{mmHg}$ & $81(79-84)$ & $82(80-84)$ & $84(81-86)$ & $\mathrm{F}=0.93, p=0.397$ \\
\hline Pulse, beats per minute & $73(71-75)$ & $75(73-77)$ & $75(73-77)$ & $\mathrm{F}=0.86, p=0.426$ \\
\hline BMI, $\mathrm{kg} / \mathrm{m}^{2}$ & $28.6(27.6-29.6)$ & $27.5(26.6-28.4)$ & $26.9(25.9-28.0)$ & $\mathrm{F}=2.46, p=0.087$ \\
\hline $\mathrm{WC}, \mathrm{cm}$ & $95.9(93.2-98.7)$ & $93.8(91.3-96.3)$ & $93.7(90.8-96.6)$ & $\mathrm{F}=0.77, p=0.463$ \\
\hline $\mathrm{WC} / \mathrm{HC}$ & $0.93(0.91-0.95)$ & $0.93(0.91-0.94)$ & $0.94(0.92-0.95)$ & $\mathrm{F}=0.30, p=0.783$ \\
\hline Hand-grip dynamometry, $\mathrm{kg}$ & $45.3(43.7-46.9)$ & $43.6(42.2-45.1)$ & $41.3(39.6-42.9)^{* *}$ & $\mathrm{~F}=5.36, p<0.005$ \\
\hline $\mathrm{TCH}, \mathrm{mmol} / \mathrm{L}$ & $5.7(5.5-6.0)$ & $5.7(5.5-5.9)$ & $5.8(5.5-6.0)$ & $\mathrm{F}=0.10, p=0.909$ \\
\hline $\mathrm{HDL} \mathrm{CH}, \mathrm{mmol} / \mathrm{L}$ & $0.96(0.89-1.03)$ & $0.99(0.93-1.05)$ & $1.06(0.99-1.13)$ & $\mathrm{F}=1.89, p=0.153$ \\
\hline $\mathrm{LDL} \mathrm{CH}, \mathrm{mmol} / \mathrm{L}$ & $4.1(3.9-4.4)$ & $4.0(3.8-4.3)$ & $4.0(3.8-4.3)$ & $\mathrm{F}=0.22, p=0.801$ \\
\hline $\mathrm{TG}, \mathrm{mmol} / \mathrm{L}$ & $1.4(1.2-1.6)$ & $1.5(1.3-1.6)$ & $1.5(1.3-1.7)$ & $\mathrm{F}=0.39, p=0.680$ \\
\hline $\begin{array}{l}\text { Number of smoked } \\
\text { cigarettes per day }\end{array}$ & $15(12-18)$ & $18(15-20)$ & $18(15-21)$ & $\mathrm{F}=1.33, p=0.268$ \\
\hline $\begin{array}{l}\text { The amount of consumed } \\
\text { ethanol, g per week }\end{array}$ & $70.3(23.3-117.3)$ & 119.5 (77.3-161.7) & $209.1(160.3-257.9)^{* * *}+$ & $\mathrm{F}=7.47, p<0.001$ \\
\hline $\begin{array}{c}\text { Physical training and sports, } \\
\text { hours per week }\end{array}$ & $2.5(1.9-3.1)$ & $2.5(2.0-3.1)$ & $1.0(0.7-1.9) *++$ & $\mathrm{F}=4.86, p=0.008$ \\
\hline $\begin{array}{l}\text { Sedentary behavior, hours } \\
\text { per day }\end{array}$ & $6.4(5.7-7.2)$ & $7.8(7.2-8.5)^{* *}$ & $7.2(6.5-7.9)$ & $\mathrm{F}=4.23, p=0.015$ \\
\hline
\end{tabular}

Data are presented as M (95\% confidence interval). CVD—cardiovascular diseases, SBP—systolic blood pressure, DBP—diastolic blood pressure, BMI-body mass index, WC-waist circumference, HC - hip circumference, TCH—total cholesterol, HDL CH-high density lipoprotein cholesterol, LDL CH-low density lipoprotein cholesterol, TG—triglycerides. ${ }^{*} p<0.05,{ }^{* *} p<0.01,{ }^{* * *} p<0.001$ (versus level 1); $+p<0.05,+\dagger p<0.001$ (versus level 2). $p$-values are derived from ANOVA with post hoc comparisons using the Holm-Bonferroni method.

The comparative analysis of lifestyle and quality of life indicators in groups of men with different VE levels (Table 4) exhibited the existence of statistically highly significant inverse linear relationships between the group means for most of the studied variables and ordered VE levels: i.e., with an increase in VE level, the values of the studied indicators were declining. The greatest decrease in the studied parameters was observed in the group of men with a high VE level. 
Table 4. Comparative analysis of indicators of psychosocial environment and lifestyle in groups of males with different vital exhaustion levels.

\begin{tabular}{|c|c|c|c|c|}
\hline \multirow{2}{*}{ Studied Indicators } & \multicolumn{3}{|c|}{ Vital Exhaustion Levels } & \multirow{2}{*}{ F-Test } \\
\hline & $1(n=97)$ & $2(n=109)$ & $3(n=93)$ & \\
\hline Education & $3.4(3.2-3.5)$ & $3.2(3.0-3.4)$ & $3.2(3.0-3.4)$ & $\mathrm{F}=1.74, p=0.188$ \\
\hline Social satisfaction & $71.5(68.3-74.7)$ & $64.5(61.2-67.9) *$ & $51.8(46.7-56.8)^{* *+\dagger}$ & $\mathrm{F}=48.51, p<0.001$ \\
\hline The type of work & $3.6(3.5-3.8)$ & $3.5(3.3-3.7)$ & $3.3(3.1-3.5) *$ & $\mathrm{~F}=7.26, p=0.007$ \\
\hline Working conditions & $69.8(66.0-73.5)$ & $61.8(56.8-66.8) *$ & $57.5(52.5-62.5)^{* * *}$ & $\mathrm{~F}=13.40, p<0.001$ \\
\hline Working hours & $8.5(7.9-9.1)$ & $8.7(8.0-9.5)$ & $8.8(8.0-9.5)$ & $\mathrm{F}=0.29, p=0.588$ \\
\hline $\begin{array}{l}\text { Working relationship with } \\
\text { managers }\end{array}$ & $80.5(77.3-83.8)$ & $76.5(73.0-80.0)$ & $66.2(61.1-71.2) * *+\dagger$ & $\mathrm{F}=25.95, p<0.001$ \\
\hline $\begin{array}{l}\text { Working relationships with } \\
\text { colleagues }\end{array}$ & $83.9(81.4-86.4)$ & $80.9(78.1-83.8)$ & $72.7(68.7-76.7)^{* *+\dagger}$ & $\mathrm{F}=24.12, p<0.001$ \\
\hline Job satisfaction & $76.8(73.5-80.1)$ & $68.5(64.8-72.1)^{* *}$ & $54.7(49.8-59.6){ }^{* *}++$ & $\mathrm{F}=59.08, p<0.001$ \\
\hline Lack of stress in the workplace & $59.4(55.0-63.7)$ & $49.0(44.5-53.4)^{* *}$ & $46.5(41.6-51.4)^{* *}$ & $\mathrm{~F}=15.45, p<0.001$ \\
\hline Spiritual needs & $62.3(58.8-65.8)$ & $52.4(48.3-56.5) * *$ & $46.7(42.2-51.3)^{* *}+$ & $\mathrm{F}=27.82, p<0.001$ \\
\hline Hobby & $61.1(55.7-66.4)$ & $58.9(54.0-63.7)$ & $48.0(42.5-53.6){ }^{* *}++$ & $\mathrm{F}=11.57, p=0.001$ \\
\hline Personal happiness level & $77.1(74.2-80.1)$ & $71.1(68.1-74.1)^{* *}$ & $56.9(53.1-60.7) * *++$ & $\mathrm{F}=72.82, p<0.001$ \\
\hline Faith & $56.8(49.9-63.7)$ & $57.7(52.0-63.5)$ & $59.2(53.0-65.5)$ & $\mathrm{F}=0.28, p=0.595$ \\
\hline Enough friends & $74.9(70.5-79.3)$ & $70.7(66.2-75.3)$ & $56.7(50.8-62.6)^{* *}+\dagger$ & $\mathrm{F}=25.02, p<0.001$ \\
\hline Satisfaction with friends & $76.1(71.6-80.5)$ & $72.2(67.7-76.6)$ & $61.3(55.7-67.0)^{* *+\dagger}$ & $\mathrm{F}=17.24, p<0.001$ \\
\hline Family and relatives support & 90.7 (87.9-93.5) & $87.7(84.4-91.0)$ & $81.1(75.6-86.6) * *+$ & $\mathrm{F}=10.92, p=0.001$ \\
\hline Friends' support & $78.9(74.4-83.3)$ & $70.7(65.9-75.5)$ & $56.5(50.2-62.8)^{* * *+十+}$ & $\mathrm{F}=34.62, p<0.001$ \\
\hline $\begin{array}{c}\text { Satisfaction with the } \\
\text { authorities }\end{array}$ & $51.4(46.4-56.4)$ & $46.6(42.2-51.0)$ & $40.8(35.4-46.2)^{* *}$ & $\mathrm{~F}=8.75, p=0.003$ \\
\hline Availability of essential food & $82.4(79.1-85.7)$ & $77.2(73.8-80.6) *$ & $68.8(64.3-73.4)^{* *}+\dagger$ & $\mathrm{F}=24.78, p<0.001$ \\
\hline Personal safety & $67.9(64.5-71.3)$ & $64.3(60.8-67.8)$ & $56.5(52.2-60.8)^{* *++}$ & $\mathrm{F}=17.49, p<0.001$ \\
\hline Living space, sq. m & $75.2(64.4-86.0)$ & $76.9(63.1-90.7)$ & $56.9(51.6-62.3) *+$ & $\mathrm{F}=5.03, p=0.026$ \\
\hline $\begin{array}{l}\text { Material well-being of the } \\
\text { household }\end{array}$ & $67.2(63.9-70.6)$ & $60.1(56.6-63.6)^{* *}$ & $53.5(49.9-57.0)^{* *+\dagger}$ & $\mathrm{F}=29.62, p<0.001$ \\
\hline Satisfaction with earnings & $61.6(57.1-66.1)$ & $52.5(47.6-57.4) *$ & $47.2(42.0-52.3) * *$ & $\mathrm{~F}=16.89, p<0.001$ \\
\hline $\begin{array}{l}\text { Satisfaction with housing } \\
\text { conditions }\end{array}$ & $68.7(64.2-73.2)$ & $65.0(60.1-69.8)$ & $59.0(53.3-64.6) *$ & $\mathrm{~F}=7.10, p=0.008$ \\
\hline Environmental satisfaction & $64.0(59.6-68.4)$ & $63.9(59.9-67.8)$ & $47.7(42.9-52.5)^{* *+\dagger}$ & $\mathrm{F}=25.65, p<0.001$ \\
\hline $\begin{array}{c}\text { Satisfaction with living } \\
\text { conditions }\end{array}$ & $76.7(73.3-80.2)$ & $72.9(69.3-76.5)$ & $65.9(61.6-70.1)^{* * *}+$ & $\mathrm{F}=15.80, p<0.001$ \\
\hline Dietary diversity & $74.1(70.8-77.5)$ & $69.2(65.8-72.5)$ & $59.5(55.4-63.6)^{* *}+\dagger$ & $\mathrm{F}=30.62, p<0.001$ \\
\hline Satisfaction with food intake & $90.2(87.6-92.8)$ & 88.7 (85.9-91.5) & $79.6(75.5-83.6)^{* *+十}$ & $\mathrm{~F}=21.07, p<0.001$ \\
\hline Intimacy issues & $83.0(79.7-86.2)$ & $75.6(71.4-79.9) *$ & $64.5(59.1-69.8)^{* *+\dagger}$ & $\mathrm{F}=34.18, p<0.001$ \\
\hline Satisfaction with sleep & $76.5(72.7-80.2)$ & $65.9(61.2-70.7)^{* *}$ & $53.2(47.6-58.7)^{* *+十}$ & $\mathrm{~F}=45.47, p<0.001$ \\
\hline Family happiness & $82.3(78.5-86.2)$ & $76.8(72.8-80.9)$ & $68.6(63.3-73.8)^{* *}+$ & $\mathrm{F}=19.22, p<0.001$ \\
\hline
\end{tabular}

Data are presented as M (95\% confidence interval). ${ }^{*} p<0.05,{ }^{* *} p<0.01,{ }^{* * *} p<0.001$ (versus level 1$) ;+p<0.05,+\dagger p<0.01$, ††+ $p<0.001$ (versus level 2). $p$-values are derived from ANOVA with post hoc comparisons using the Holm-Bonferroni method.

Taking into account the close relationship between VE and PS (Table 2), we corrected intergroup differences in quality of life and lifestyle indicators for the PS level, which did not significantly affect previously identified (Table 4) inverse linear trends and intergroup differences for most of the studied indicators (Table 5). The exceptions were the lack of stress in the workplace, satisfaction with the authorities, personal safety, and satisfaction with housing conditions, the VE effect that has reduced to statistically insignificant values (Table 5). 
Table 5. Comparative analysis of indicators of psychosocial environment and lifestyle in groups of males with different vital exhaustion levels after the correction for the psychosocial stress score.

\begin{tabular}{|c|c|c|c|c|}
\hline \multirow{2}{*}{ Studied Indicators } & \multicolumn{3}{|c|}{ Vital Exhaustion Levels } & \multirow{2}{*}{ F-Test } \\
\hline & $1(n=97)$ & $2(n=109)$ & $3(n=93)$ & \\
\hline Education & $3.4(3.2-3.6)$ & $3.2(3.0-3.4)$ & $3.1(2.9-3.4)$ & $\mathrm{F}=1.29, p=0.276$ \\
\hline Social satisfaction & $70.9(66.7-75.0)$ & $64.4(60.7-68.2) *$ & $52.5(48.2-56.7) * * *++\dagger$ & $\mathrm{F}=17.12, p<0.001$ \\
\hline The type of work & $3.6(3.5-3.8)$ & $3.5(3.3-3.7)$ & $3.2(3.1-3.4) *$ & $\mathrm{~F}=3.89, p=0.021$ \\
\hline Working conditions & $68.5(63.7-73.2)$ & $61.8(57.4-66.2)$ & $59.2(53.9-64.5) *$ & $\mathrm{~F}=3.38, p=0.035$ \\
\hline Working hours & $8.7(7.9-9.4)$ & $8.8(8.1-9.5)$ & $8.5(7.7-9.3)$ & $\mathrm{F}=0.18, p=0.837$ \\
\hline $\begin{array}{c}\text { Working relationship with } \\
\text { managers }\end{array}$ & $80.3(76.4-84.3)$ & $76.5(72.7-80.3)$ & $66.4(62.1-70.7)^{* * *+十}$ & $\mathrm{~F}=10.41, p<0.001$ \\
\hline $\begin{array}{l}\text { Working relationships with } \\
\text { colleagues }\end{array}$ & $82.4(79.2-85.5)$ & $80.8(77.9-83.7)$ & $74.6(71.2-78.1)^{* *}+$ & $\mathrm{F}=5.39, p=0.005$ \\
\hline Job satisfaction & $75.7(71.6-79.7)$ & $68.5(64.7-72.2) *$ & $56.1(51.7-60.5)^{* * *++\dagger}$ & $\mathrm{F}=18.90, p<0.001$ \\
\hline Lack of stress in the workplace & $55.5(51.1-60.0)$ & $48.7(44.6-52.9)$ & $51.3(46.4-56.2)$ & $\mathrm{F}=2.41, p=0.091$ \\
\hline Spiritual needs & $62.7(58.4-67.0)$ & $52.6(48.7-56.4)^{* *}$ & $46.3(41.8-50.7)^{* * *} \dagger$ & $\mathrm{F}=12.61, p<0.001$ \\
\hline Hobby & $60.3(54.8-65.9)$ & $58.6(53.6-63.6)$ & $48.8(43.1-54.6) *+$ & $\mathrm{F}=4.27, p=0.015$ \\
\hline Personal happiness level & $76.2(72.8-79.6)$ & $71.0(67.9-74.1) *$ & $58.0(54.4-61.6)^{* * *+十+}$ & $\mathrm{F}=25.04, p<0.001$ \\
\hline Faith & $56.1(49.4-62.7)$ & $57.6(51.6-63.7)$ & $60.1(53.2-67.0)$ & $\mathrm{F}=0.31, p=0.734$ \\
\hline Enough friends & $73.7(68.5-78.8)$ & $71.3(66.6-76.0)$ & $58.2(52.8-63.5)^{* * *+十+}$ & $\mathrm{F}=8.92, p<0.001$ \\
\hline Satisfaction with friends & $74.7(69.6-79.8)$ & $72.3(67.7-76.9)$ & $62.9(57.6-68.2) * *+$ & $\mathrm{F}=5.03, p=0.007$ \\
\hline Family and relatives support & $90.1(85.9-94.3)$ & $87.5(83.8-91.3)$ & $81.8(77.5-86.2) *$ & $\mathrm{~F}=3.40, p=0.035$ \\
\hline Friends' support & $77.9(72.3-83.4)$ & $70.8(65.8-75.8)$ & $57.6(51.9-63.3)^{* * *}+\dagger$ & $\mathrm{F}=11.64, p<0.001$ \\
\hline $\begin{array}{c}\text { Satisfaction with the } \\
\text { authorities }\end{array}$ & $47.3(42.3-52.3)$ & $46.2(41.7-50.7)$ & $45.5(40.3-50.6)$ & $\mathrm{F}=0.12, p=0.887$ \\
\hline Availability of essential food & $82.8(78.9-86.8)$ & $77.5(73.9-81.1) *$ & $68.3(64.3-72.4) * * *+\dagger$ & $\mathrm{F}=11.45, p<0.001$ \\
\hline Personal safety & $65.6(61.7-69.5)$ & $64.1(60.6-67.6)$ & $59.1(55.1-63.1)$ & $\mathrm{F}=2.61, p=0.076$ \\
\hline Living space, sq. m & $77.3(65.9-88.7)$ & $77.5(66.8-88.1)$ & $54.4(42.2-66.7)^{*}+$ & $\mathrm{F}=4.48, p=0.012$ \\
\hline $\begin{array}{l}\text { Material well-being of the } \\
\text { household }\end{array}$ & $66.7(63.1-70.4)$ & $60.3(56.9-63.6) *$ & $54.0(50.2-57.9)^{* *}+$ & $\mathrm{F}=9.94, p<0.001$ \\
\hline Satisfaction with earnings & $61.9(56.8-67.1)$ & $53.0(48.4-57.6) *$ & $46.8(41.5-52.1)^{* * *}$ & $\mathrm{~F}=7.50, p<0.001$ \\
\hline $\begin{array}{l}\text { Satisfaction with housing } \\
\text { conditions }\end{array}$ & $68.2(62.9-73.5)$ & $64.6(59.8-69.4)$ & $59.5(54.1-65.0)$ & $\mathrm{F}=2.25, p=0.107$ \\
\hline Environmental satisfaction & $62.0(57.4-66.6)$ & $63.5(59.4-67.7)$ & $50.0(45.2-54.8))^{* *}+\dagger$ & $\mathrm{F}=9.42, p<0.001$ \\
\hline $\begin{array}{c}\text { Satisfaction with living } \\
\text { conditions }\end{array}$ & $76.9(73.0-80.8)$ & $73.5(70.0-77.0)$ & $65.7(61.6-69.7)^{* * *++}$ & $\mathrm{F}=7.28, p<0.001$ \\
\hline Dietary diversity & $75.0(71.2-78.9)$ & $69.2(65.7-72.6)$ * & $58.5(54.5-62.4)^{* * *+十+}$ & $\mathrm{F}=16.04, p<0.001$ \\
\hline Satisfaction with food intake & $90.0(86.6-93.3)$ & $88.7(85.7-91.8)$ & $79.9(76.4-83.3)^{* * *+十+~}$ & $\mathrm{~F}=9.26, p<0.001$ \\
\hline Intimacy issues & $82.7(78.1-87.2)$ & $76.3(72.2-80.4)$ * & $64.9(60.2-69.5)^{* * *++\dagger}$ & $\mathrm{F}=13.30, p<0.001$ \\
\hline Satisfaction with sleep & $74.1(69.1-79.1)$ & $65.6(61.1-70.1) *$ & $55.9(50.8-61.1)^{* * *}+$ & $\mathrm{F}=11.19, p<0.001$ \\
\hline Family happiness & $80.7(76.3-85.0)$ & $77.0(72.9-81.1)$ & $70.6(65.7-75.5) *$ & $\mathrm{~F}=4.04, p=0.019$ \\
\hline
\end{tabular}

Data are presented as M (95\% confidence interval). ${ }^{*} p<0.05,{ }^{* *} p<0.01,{ }^{* * *} p<0.001$ (versus level 1$) ;+p<0.05,+\dagger p<0.01$, ††+ $p<0.001$ (versus level 2). $p$-values are derived from ANOVA with post hoc comparisons using the Holm-Bonferroni method.

Multiple linear regression was used to assess the relationship between VE and the studied parameters. Initially, 27 independent variables, characterizing the lifestyle and quality of life, and statistically significantly correlating with VE, were introduced into the regression model. In the final model, as a result of stepwise selection, just eight independent variables remained (Table 6). We evaluated the regression model as consistent and explanatory, since the number of used observations was much greater than the number of independent variables in the model. The multiple correlation coefficient was statistically significant $(\mathrm{R}=0.695 ; p=0.049)$, the regression coefficients were also significant, and VIF values implied the absence of multicollinearity (Table 6). Negative values of regression coefficients (B and beta) for PS implied a directly proportional relationship with VE caused by inversely proportional values of the scales for PS and VE. The VE level was directly proportional to the PS level and inversely proportional to other explanatory variables, with the exception of the amount of consumed alcohol (ethanol). The greatest contribution to VE was made by the personal happiness level, explaining $25.5 \%$ of its variability. The pro- 
portion of the VE variance uniquely attributable to PS was just $9.3 \%$. Further, in decreasing order of the explained variance proportion are: the employment satisfaction, sleep satisfaction, total amount of consumed ethanol, muscle strength (hand-grip dynamometry), living conditions in the residential neighborhood, and spiritual needs. Altogether, explanatory variables accounted for $46.6 \%$ of the variability in VE levels.

Table 6. Multiple linear regression of vital exhaustion association with indicators of psychosocial environment and lifestyle in 41-44-year-old men.

\begin{tabular}{|c|c|c|c|c|c|c|}
\hline Independent Variables & B $(95 \%$ CI $)$ & $p$ & $\mathbf{b}$ & $\mathbf{R}^{2}$ (Partial) & $R^{2}$ (Adjusted) & VIF \\
\hline Constant term & $19.140(16.546 ; 21.733)$ & $<0.001$ & - & - & & - \\
\hline Personal happiness level & $-0.036(-0.056 ;-0.015)$ & 0.001 & -0.193 & 0.255 & & 1.523 \\
\hline Psychosocial stress & $-1.494(-2.097 ;-0.891)$ & $<0.001$ & -0.247 & 0.093 & & 1.175 \\
\hline Job satisfaction & $-0.027(-0.045 ;-0.010)$ & 0.002 & -0.171 & 0.049 & & 1.423 \\
\hline Satisfaction with sleep & $-0.023(-0.036 ;-0.009)$ & 0.001 & -0.177 & 0.028 & 0.466 & 1.258 \\
\hline $\begin{array}{l}\text { Total volume of consumed } \\
\text { ethanol, g per week }\end{array}$ & $0.002(0.001 ; 0.003)$ & 0.001 & 0.154 & 0.023 & & 1.045 \\
\hline Hand-grip dynamometry & $-0.060(-0.101 ;-0.019)$ & 0.001 & -0.138 & 0.016 & & 1.045 \\
\hline $\begin{array}{l}\text { Satisfaction with living } \\
\text { conditions }\end{array}$ & $-0.020(-0.037 ;-0.003)$ & 0.023 & -0.113 & 0.011 & & 1.118 \\
\hline Spiritual needs & $-0.016(-0.031 ;-0.0001)$ & 0.049 & -0.097 & 0.008 & & 1.103 \\
\hline
\end{tabular}

Dependent variable: psychosocial stress score. B—regression coefficient; CI—confidence interval; $p$-value—the probability of obtaining results at least as extreme as the observed results of a statistical hypothesis test, assuming that the null hypothesis is correct; $b$ (beta) standardized regression coefficient; $\mathrm{R}^{2}$ — coefficient of multiple determination, VIF—variance inflation factor.

\section{Discussion}

Our study subjects were represented by men selected for subsequent analysis at their seventh visit to a physician within the framework of the 32-year prospective study.

The results of our research showed that the VE level in 41-44-year-old men was associated with excessive alcohol consumption and increased likelihood of developing $\mathrm{AH}$.

Additionally, the VE level in 41-44-year-old men, regardless of their PS score, was associated with an increase in the amount of consumed alcohol and duration of sedentary behavior, along with reduction in their physical activity and muscle strength. The linear association of VE with BMI and HDL cholesterol was caused by the confounding effect of the PS.

An increase in the VE level in 41-44-year-old men was associated with a decrease in most indicators of quality of life and lifestyle after their adjustment for the PS score. The linear relationships between VE and lack of stress in the workplace, satisfaction with the authorities, personal safety, and satisfaction with housing conditions were also caused by the confounding effect of the PS.

The most significant determinants of the VE in 41-44-year-old men were personal happiness level, PS score, satisfaction with employment and sleep, total amount of consumed ethanol, muscle strength, living conditions in the residential neighborhood, and spiritual needs.

The results of our study suggested that over $25 \%$ of surveyed men had a medium VE level, while every tenth study subject had a critically high VE value. Our findings partially agree with the results of the study conducted in Siberia on Tyumen men in terms of the critical VE level (12.3\%), but are lower than the fraction of Tyumen males with a medium VE level (38.6\%) [23], and are even lower than the level of corresponding indicators revealed by some studies conducted in Europe, where the proportion of men with a medium VE level was $51.7 \%$ and with a critical high VE level was 31.5\% [24]. The lower prevalence of VE in the Moscow area, as compared to the Tyumen region, may have been caused by the higher quality of life in Muscovites and more severe climatic conditions in the Siberian region. We hypothesize that a higher level of VE in the male population of the Netherlands, compared with Moscow men, could be influenced by a more intense workload associated 
with their professional activities, an insufficient social circle, and also, possibly, by the nonexistent or weak social support from relatives and friends.

\subsection{Vital Exhaustion versus Cardiovascular Risk Factors}

The direct dependence of AH frequency on VE level that we have established in men was consistent with the results of numerous foreign and domestic studies, which drew attention to the significant contribution of VE to high systolic blood pressure, coronary artery disease, and development of cardiovascular events $[2,6,7,10,11,25,26]$. Van Diest et al. and Balog et al. proposed that VE could represent a more somatic dimension of distress $[4,27]$. According to the Hungarian study, VE was the only independent, significant, and reliable psychological predictor of the recurrence of vascular events [26].

The lack of relationship between the VE level and BP in our study was apparently caused by the fact that the majority of males with $\mathrm{AH}(71.1 \%)$ received antihypertensive medicamentous therapy, which was reflected in their average systolic and diastolic BP measurements in all groups.

The relationship among the VE level and excessive alcohol consumption revealed in our study was consistent with other studies that established direct relationships between the VE and total amount of consumed ethanol, sedentary lifestyle, and smoking [2,28,29]. These dependences seem very predictable, since men with high VE are more likely to offset the frustration and demoralization, inherent in VE, by choosing the fastest and seemingly most affordable way to correct this situation by means of the alcohol abuse. Excessive fatigue and weakness, subjectively experienced by men, were also represented in our study by entirely objective characteristics: a reduction in hand-grip dynamometry and an increase in sedentary behavior with higher VE levels. However, despite multiple studies showing an increase in the frequency of smoking with an increase in the VE level in various population groups $[2,28,29]$, we did not encounter such relationship. Perhaps, this was due to insufficient number of observations.

The 2008 study by Bryant et al. [30] and 2011 study by Igna et al. [28] revealed the direct dependence of BMI on the VE level, which was not supported by the 2012 study of Iversen et al. [31]. Our research demonstrated an inverse relationship of the kind: specifically, higher VE corresponded to lower BMI values. Enlarged HDL cholesterol content with an increase of $\mathrm{VE}$, also demonstrated in our research, was not consistent with the results of the study by Koertge et al. [32], in which high VE was associated with an enlarged level of HDL cholesterol. Hence, we conclude that the established association of VE with BMI and HDL cholesterol was caused by the confounding effect of PS.

We think that the strongest established relationship between the levels of VE and PS was quite expected; this finding has supported the 2008 study by Bellingrath et al., who demonstrated that VE could be viewed as a consequence of prolonged stress and lack of resources required to adapt to the stress [33]. Men with increased VE were spending shorter time on hobbies and spiritual needs, which has indicated a serious deterioration in their quality of life and impoverishment of their lifestyle. The latter was expressed in demoralization, loss of strength, and excessive fatigue. Hobbies and spiritual needs of a person are protective resources or coping strategies aimed, among other things, at overcoming psychoemotional stress [34].

\subsection{Vital Exhaustion versus Sociopsychological Environment, Lifestyle and Quality of Life}

Since VE is an indicator of both physical and psychological well-being, a noticeable decline in the quality of life and lifestyle impoverishment in men with increases in VE can be traced for nearly all studied indicators. It is important to note that correction for the PS score did not affect previously identified trends and intergroup differences for most of the studied indicators, with the exception of the following: lack of stress in the workplace, satisfaction with the authorities, personal safety, and satisfaction with housing conditions. This finding implies that the primary reason for the relationship between a high level of VE and these indicators is stress per se, caused by dissatisfaction with 
individual (work, housing) and social (satisfaction with the authorities, personal safety) living conditions [35] over a long period of time, which leads to physical and emotional exhaustion, once again confirming an assumption by $\mathrm{S}$. Bellingrath that VE is a consequence of prolonged stress $[8,33]$ associated in men with existing living conditions.

It should be pointed out that an aggravation of VE in men has a negative impact on the most important parts of their lives. Fatigue and loss of energy, which characterize VE, significantly affect one of the main priorities of their lives: work and professional development, which is expressed in a reduction of job satisfaction and deterioration in the type and conditions of employment. Besides, psychosocial risks associated with work are among the most challenging issues of occupational health and work safety that can significantly affect the physical and mental health of employees and have a negative impact on the performance of organizations (absenteeism, high turnover, low labor productivity) and even the entire national economy [36,37]. Interpersonal relationship issues (with family, colleagues and friends) are also aggravated with an increase in VE.

It should be emphasized that an increase in VE in men, observed in our study, was associated with changes in their eating habits, which most likely related to a somewhat significant association between VE and stress, since activation of the adrenal sympathetic cerebral systems during stress leads to the release of adrenaline and norepinephrine, which in turn may suppress appetite [38]. Hence, we could assume that appetite reduction was associated with a decrease in dietary diversity, availability, and satisfaction with the amount of consumed food. Consequently, all of these indicators, revealed with an increase in VE (Table 5), could significantly affect the eating habits, most often, impoverishing their quality and reducing dietary diversity, based on self-evaluations by the respondents. A reduction in BMI and an increase in HDL cholesterol in men with a pronounced VE, prior to the correction for the PS level, implies a confounding effect of the stress factor on the development of VE.

An increase of VE in men was associated with a decline in satisfaction experienced from the support by significant social environment (family, relatives, friends), as well as from communication with friends and position in the society. The physical and emotional conditions in men, caused by VE, considerably limited their social contacts and reduced the level of their communication, thus significantly impoverishing their quality of life. Social support from the family and friends is a strong and persistent determinant of mental health in adults, and this association has been noted in many studies [8,39]; on the contrary, social isolation may contribute to the onset of heart failure, which was shown on the general population of the United States via an increase in VE [8].

We demonstrated that PS, job satisfaction, sleep satisfaction, total volume of consumed ethanol, muscle strength, living conditions in the residential neighborhood, and spiritual needs were significant determinants of $\mathrm{VE}$, albeit to a lesser extent than the personal happiness. In contrast, in the Men Stress 40+ study [8], chronic stress was the only significant VE predictor.

\section{Conclusions}

The results of our study assessing the association of vital exhaustion with lifestyle and quality of life, as well as with risk factors for cardiovascular diseases in 41-44-year-old Muscovite men, disclosed that high level of vital exhaustion in these men was associated with such cardiovascular disease risk factors as arterial hypertension, sedentary behavior, and excessive alcohol consumption and lower values of most indicators of lifestyle and quality of life.

The most significant determinants of the vital exhaustion in 41-44-year-old men were personal happiness, psychosocial stress, job satisfaction, sleep satisfaction, total volume of consumed ethanol per unit time, muscle strength, living conditions in a residential neighborhood, and spiritual needs. All of these need to be taken into account, along with other behavioral and psychosocial risk factors for cardiovascular diseases, when developing the programs aimed at strengthening and maintaining the population health. 
An important implication of our research is that additional studies are needed to extend our findings to women, and men of other ages and from other regions of Russia.

\section{Study Strengths and Limitations}

Our research was carried out on a homogeneous group (in terms of age and gender) living in similar conditions. In Russia, there have been no similar studies examining the associations of vital exhaustion with risk factors for cardiovascular diseases, quality of life and lifestyle. In the available worldwide scientific publications, such studies are also extremely rare and are mainly devoted to individual aspects of the problem under study.

However, this study is not without its drawbacks. A significant limitation of the study is the low response rate of the participants, compared with the original study. The reasons for this fact could be significant socioeconomic and political changes in the country that have occurred over 32 years. As a result, manystudy subjects surveyed in 1984 (as 11-yearold school students) changed their place of residence; hence, their contact information was no more available. Besides, high male mortality (for various reasons) in the 1990s in Russia was another negative factor. Unfortunately, it is not possible to estimate how low response rate could have influenced our results due to the lack of any regularities in the 'contact information loss'. However, we do not repudiate that the low response rate could have affected the obtained results. In fact, the conducted study was cross-sectional in its design, which did not allow evaluating the results in terms of cause-and-effect relationships. In addition, it should be noted that some of the parameters under study were quantitively assessed using the questionnaires-i.e., their values could only be true given the assumption of honest responses.

Additionally, one of the limitations of our study is the fact that some of the questionnaires we used were not validated.

Author Contributions: Conceptualization, M.B.K., V.B.R. and O.M.D.; methodology, M.B.K., V.B.R.; software, V.B.R. and S.A.M.; validation, M.B.K., V.B.R. and S.A.M.; formal analysis, M.B.K., V.B.R. and S.A.M.; investigation, M.B.K.; resources, O.M.D.; data curation, M.B.K., V.B.R. and O.M.D.; writing—original draft preparation, M.B.K., V.B.R. and A.R.K.; writing—review and editing, M.B.K., A.R.K. and S.A.M.; visualization, M.B.K.; supervision, O.M.D.; project administration, M.B.K. and O.M.D.; funding acquisition, O.M.D. All authors have read and agreed to the published version of the manuscript.

Funding: This research received no external funding.

Institutional Review Board Statement: The study was conducted according to the guidelines of the Declaration of Helsinki, and approved by the Ethics Committee of National Medical Research Center for Therapy and Preventive Medicine, Moscow, Russia (protocol number 07-03/12, 3 July 2012).

Informed Consent Statement: Informed consent was obtained from all subjects involved in the study.

Data Availability Statement: The datasets analysed during the current study are not publicly available due to the privacy of individuals that participated in the study but are available from the corresponding author on reasonable request.

Conflicts of Interest: The authors declare no conflict of interest.

\section{References}

1. European guidelines on cardiovascular disease prevention in clinical practice (2016 revision). Russ. J. Cardiol. 2017, 22, 7-85. [CrossRef]

2. Cohen, R.; Bavishi, C.; Haider, S.; Thankachen, J.; Rozanski, A. Meta-analysis of relation of vital exhaustion to cardiovascular disease events. Am. J. Cardiol. 2017, 119, 1211-1216. [CrossRef]

3. Appels, A.; Hoppener, P.; Mulder, P. A questionnaire to assess premonitory symptoms of myocardial infarction. Int. J. Cardiol. 1987, 17, 15-24. [CrossRef]

4. Balog, P.; Falger, P.R.J.; Szabó, G.; Rafael, B.; Székely, A.; Thege, B.K. Are vital exhaustion and depression independent risk factors for cardiovascular disease morbidity? Health Psychol. 2017, 36, 740-748. [CrossRef] 
5. Gafarov, V.; Voevoda, M.; Gromova, E.; Maksimov, V.; Gagulin, I.; Yudin, N.; Gafarova, A.; Mishakova, T. Cardiovascular diseases and vital exhaustion: Longitudinal study in Russia/Siberia (WHO MONICA—psychosocial program). Russ. J. Cardiol. 2016, 4, 115-123. [CrossRef]

6. Frestad, D.; Prescott, E. Vital exhaustion and coronary heart disease risk: A systematic review and meta-analysis. Psychosom. Med. 2017, 79, 260-272. [CrossRef]

7. Batelaan, N.M.; Seldenrijk, A.; Bot, M.; Van Balkom, A.J.L.M.; Penninx, B.W.J.H. Anxiety and new onset of cardiovascular disease: Critical review and meta-analysis. Br. J. Psychiatry 2016, 208, 223. [CrossRef] [PubMed]

8. Noser, E.; Fischer, S.; Ruppen, J.; Ehlert, U. Psychobiological stress in vital exhaustion. Findings from the Men Stress 40+ study. J. Psychosom. Res. 2018, 105, 14-20. [CrossRef] [PubMed]

9. Jousilahti, P.; Vartiainen, E.; Tuomilehto, J.; Puska, P. Sex, age, cardiovascular risk factors, and coronary heart disease: A prospective follow-up study of 14786 middle-aged men and women in Finland. Circulation 1999, 99, 1165-1172. [CrossRef]

10. Schnohr, P.; Marott, J.L.; Kristensen, T.S.; Gyntelberg, F.; Gronbaek, M.; Lange, P.; Jensen, M.T.; Jensen, G.B.; Prescott, E. Ranking of psychosocial and traditional risk factors by importance for coronary heart disease: The Copenhagen City Heart Study. Eur. Heart J. 2015, 36, 1385. [CrossRef] [PubMed]

11. Meyer, T.; Hussein, S.; Lange, H.W.; Herrmann-Lingen, C. Anxiety is associated with a reduction in both mortality and major adverse cardiovascular events five years after coronary stenting. Eur. J. Prev. Cardiol. 2015, 22, 75-82. [CrossRef]

12. Liu, B.; Floud, S.; Pirie, K.; Green, J.; Peto, R.; Beral, V. Does happiness itself directly affect mortality? The prospective UK Million Women Study. Lancet 2016, 387, 874-881. [CrossRef]

13. Diest, R.V.; Appels, A. Vital exhaustion: Behavioural and biological correlates. Curr. Opin. Psychiatry 2002, 15, 639-641. [CrossRef]

14. Cohen, B.E.; Edmondson, D.; Kronish, I.M. State of the Art Review: Depression, Stress, Anxiety, and Cardiovascular Disease. Am. J. Hypertens. 2015, 28, 1295-1302. [CrossRef]

15. Schoch, J.; Noser, E.; Ehlert, U. Do implicit motives influence perceived chronic stress and vital exhaustion? Front. Psychol. 2018, 9, 1149. [CrossRef]

16. National Cholesterol Education Program (US). Expert Panel on Detection, Evaluation, and Treatment of High Blood Cholesterol in Adults. Executive Summary of the Third Report of the National Cholesterol Education Program (NCEP) Expert Panel on Detection, Evaluation, and Treatment of High Blood Cholesterol in Adults (Adult Treatment Panel III). JAMA 2001, 285, $2486-2497$. [CrossRef]

17. Kopina, O.S.; Suslova, E.A.; Zaikin, E.V. Population studies of psychosocial stress as a risk factor for cardiovascular diseases. Kardiologiia 1996, 36, 53-56.

18. Gundarov, I.A. Quality of life. Sib. Health 1995, 1, 15-16.

19. Craig, C.L.; Marshall, A.L.; Sjöström, M.; Bauman, A.E.; Booth, M.L.; Ainsworth, B.E.; Pratt, M.; Ekelund, U.; Yngve, A.; Sallis, J.F.; et al. International physical activity questionnaire: 12-country reliability and validity. Med. Sci. Sports Exerc. 2003, 35, 1381-1395. [CrossRef] [PubMed]

20. Guidelines for Data Processing and Analysis of the International Physical Activity Questionnaire (IPAQ) —Short and Long Forms. 2005. Available online: http:/ / www.ipaq.ki.se (accessed on 1 July 2021).

21. Zohoori, N.; Mroz, T.A.; Popkin, B.; Glinskaya, E.; Lokshin, M.; Mancini, D.; Kozyreva, P.; Kosolapov, M.; Swafford, M. Monitoring the economic transition in the Russian Federation and its implications for the demographic crisis: The Russian Longitudinal Monitoring Survey. World Dev. 1998, 26, 1977-1993. [CrossRef]

22. Shkolnikova, M.; Shalnova, S.; Shkolnikov, V.M.; Metelskaya, V.; Deev, A.; Andreev, E.; Jdanov, D.; Vaupel, J.W. Biological mechanisms of disease and death in Moscow: Rationale and design of the Survey on Stress, Aging and Health in Russia (SAHR). BMC Public Health 2009, 9, 293. [CrossRef]

23. Akimova, E.V.; Akimov, M.J.; Gakova, E.I.; Kayumova, M.M.; Gafarov, V.V.; Kuznetsov, V.A. Levels of depression and life exhaustion in the open population of the middle urbanized Siberian city: Gender differences. Ther. Arch. 2019, 91, 48-52. [CrossRef]

24. Hoekstra, T.; Barbosa-Leiker, C.; Twisk, J.W. Vital exhaustion and markers of low-grade inflammation in healthy adults: The Amsterdam Growth and Health Longitudinal Study. Stress Health 2013, 29, 392-400. [CrossRef] [PubMed]

25. Taratukhin, E.O. The biopsychosocial approach as a novel requirement for interdisciplinarity. Russ. J. Cardiol. 2015, 20, 80-83. [CrossRef]

26. Balog, P.; KonkolÿThege, B. The role of vital exhaustion in predicting the recurrence of vascular events: A longitudinal study. Int. J. Clin. Health Psychol. 2019, 19, 75-79. [CrossRef] [PubMed]

27. Van Diest, R.; Appels, A. Vital exhaustion and depression: A conceptual study. J. Psychosom. Res. 1991, 35, 535-544. [CrossRef]

28. Igna, C.V.; Julkunen, J.; Vanhanen, H. Vital exhaustion, depressive symptoms and serum triglyceride levels in high-risk middleaged men. Psychiatry Res. 2011, 187, 363-369. [CrossRef]

29. Just-Østergaard, E.; Mortensen, E.L.; Tolstrup, J.S.; Flensborg-Madsen, T. Vital exhaustion and risk of alcohol use disorders: A prospective cohort study. J. Psychosom. Res. 2018, 114, 25-30. [CrossRef]

30. Bryant, M.J.; Stevens, J.; Truesdale, K.P.; Mosley, T.; Chambless, L. Obesity and vital exhaustion: Analysis of the atherosclerosis risk in the communities study. Obesity 2008, 16, 1545-1551. [CrossRef]

31. Iversen, L.B.; Strandberg-Larsen, K.; Prescott, E.; Schnohr, P.; Rod, N.H. Psychosocial risk factors, weight changes and risk of obesity: The Copenhagen City Heart Study. Eur. J. Epidemiol. 2012, 27, 119-130. [CrossRef] 
32. Koertge, J.C.; Ahnve, S.; Schenck-Gustafsson, K.; Orth-Gomer, K.; Wamala, S.P. Vital exhaustion in relation to lifestyle and lipid profile in healthy women. Int. J. Behav. Med. 2003, 10, 44-55. [CrossRef] [PubMed]

33. Bellingrath, S.; Weigl, T.; Kudielka, B.M. Cortisol dysregulation in school teachers in relation to burnout, vital exhaustion, and effort-reward-imbalance. Biol. Psychol. 2008, 78, 104-113. [CrossRef] [PubMed]

34. Fu, R.; Noguchi, H.; Tachikawa, H.; Aiba, M.; Nakamine, S.; Kawamura, A.; Takahashi, H.; Tamiya, N. Relation between social network and psychological distress among middle-aged adults in Japan: Evidence from a national longitudinal survey. Soc. Sci. Med. 2017, 175, 58-65. [CrossRef]

35. Kotova, M.B.; Rozanov, V.B.; Aleksandrov, A.A.; Drapkina, O.M. Association of psychosocial stress with the social environment, lifestyle and risk factors for cardiovascular diseases in middle-aged male Muscovites. Russ. J. Cardiol. 2021, 26, 4335. [CrossRef]

36. OSHwiki Contributors. Psychosocial Risks and Workers Health. 2020. Available online: http://oshwiki.eu/index.php?title= Psychosocial_risks_and_workers_health\&oldid=252881 (accessed on 1 July 2021).

37. Burr, H.; Formazin, M.; Pohrt, A. Methodological and conceptual issues regarding occupational psychosocial coronary heart disease epidemiology. Scand. J. Work Environ. Health 2016, 42, 251-255. [CrossRef] [PubMed]

38. Elliot, S.J. Psychosocial stress, women and heart health: A critical review. Soc. Sci. Med. 1995, 40, 105-115. [CrossRef]

39. Van Dyck, D.; Teychenne, M.; McNaughton, S.; De Bourdeaudhuij, I.; Salmon, J. Relationship of the perceived social and physical environment with mental health-related quality of life in middle-aged and older adults: Mediating effects of physical activity. PLoS ONE 2015, 10, e0120475. [CrossRef] [PubMed] 